\title{
A Randomized Trial of Social Worker Led Home-based Case Management to Improve Outcomes for Caregivers of Acute Stroke Patients During the Transition Period.
}

Mathew Reeves PhD, Amanda Woodward PhD, Michele Fritz MS, Paul Freddolino PhD, Constantinos Coursaris PhD, Sarah Swierenga PhD, Mojdeh Nasiri MD, Anne Hughes PhD. Department of Epidemiology and School of Social Work, Michigan State University, East Lansing, Michigan, USA.

\section{Background and Objective:}

Navigating the in-home post-stroke transition period can be associated with substantial challenges for both patients and caregivers. Few evidence-based programs currently exist that prepare and assist caregivers of stroke patients. The Michigan Stroke Transitions Trial (MISTT) tested the efficacy of a social work case management (SWCM) program - with or without access to an online website - to improve outcomes for stroke patients and their caregivers during the transition period..

\section{Trial Design and Intervention:}

MISTT is a randomized, pragmatic, open, 3-group parallel designed trial conducted in 3 Michigan hospitals. Eligible hospitalized acute stroke patients had to return home directly or within 4 weeks of entering a rehab facility. Caregivers - defined as the adult most responsible for assisting the patient - were also enrolled at the time of discharge. Participants were randomized to either:

- Group 1) Usual care, OR

- Group 2) SWCM program (8-12 week case management program delivered by trained social workers that included home visits and regular phone contact), OR

- Group 3) SWCM program plus MISTT website (an information, education, and transition support resource designed for use by both patients and caregivers).

Figure: Consort flow diagram of caregiver enrollment and follow-up

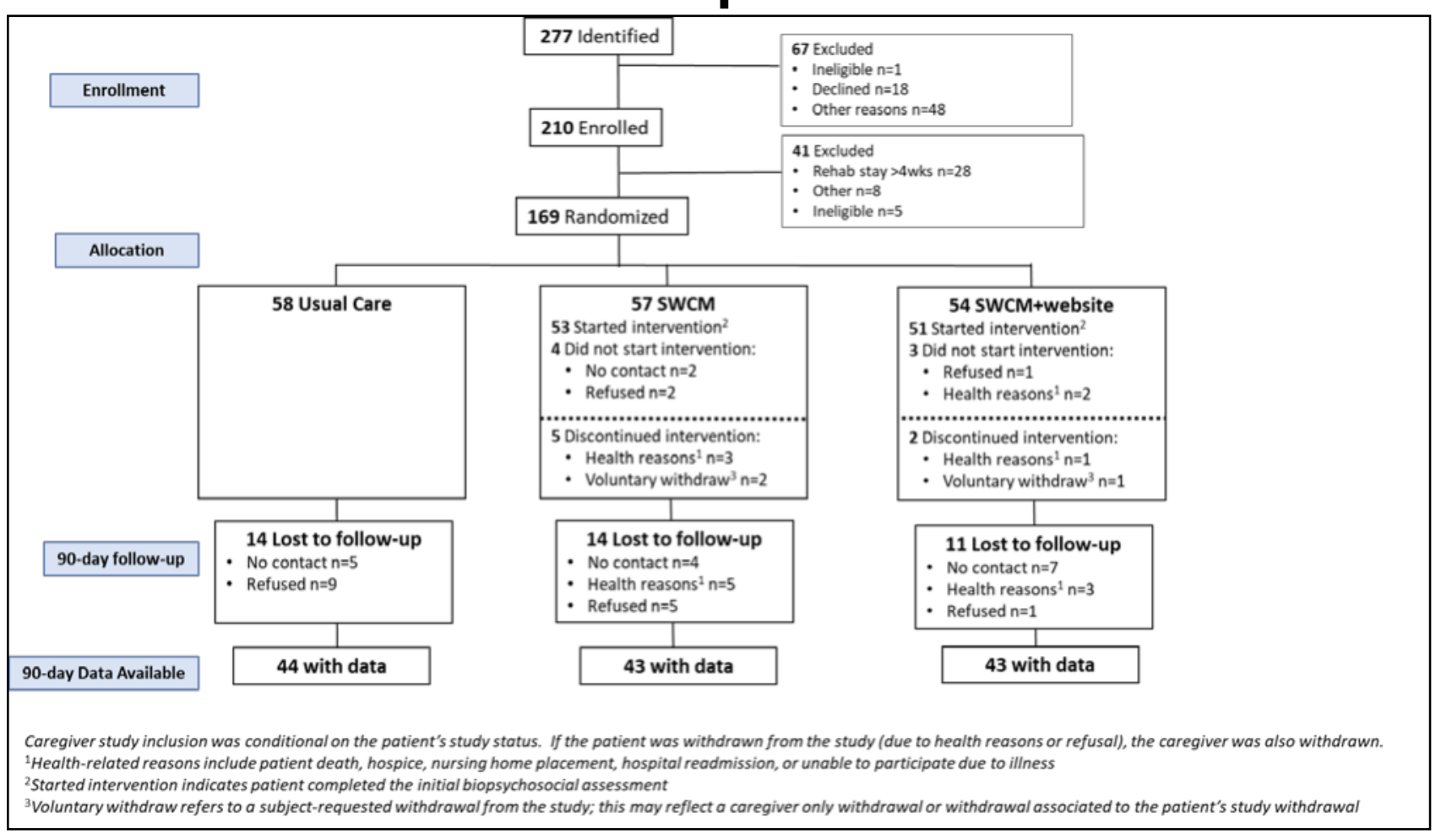

\section{Outcomes Data}

Outcomes data were collected by telephone from caregivers at 7-days and 90-days Primary caregiver outcomes were the Bakas Caregiving Outcomes Scale (BCOS) (range 15-105) and PHQ-9 (range 0-27) depression screening tool. Statistical analyses compared the mean change in scores (90-d minus 7-d) between treatment groups (differences-in-differences approach).

\section{Results:}

Among 265 randomized stroke patients, 169 (64\%) had a consented caregiver (Figure). Mean caregiver age was 58 years, $77 \%$ female, $19 \%$ non-white, $60 \%$ were spouses, and $76 \%$ lived with the patient. Mean patient age was $66,49 \%$ female, $68 \%$ mild stroke. 90 -day data were available for $130 / 169(77 \%)$ of caregivers (Figure). There were no statistically significant between group differences in the change in either the BCOS or PHQ-9 scores over the 90-day trial (Table). Changes in secondary outcomes (unhealthy days, PROMIS informational and emotional support) were also limited.

\section{Conclusions:}

An intervention that combined social worker led case management with access to an online website did not result in less stress or depression among caregivers.

Table: Group differences in caregiver outcomes (Differences-in Differences).

\begin{tabular}{|c|c|c|c|c|c|c|}
\hline \multicolumn{7}{|c|}{ Bakas Caregiver Outcomes Scale (BCOS) } \\
\hline & \multicolumn{3}{|c|}{ LS Means (SE) } & \multicolumn{3}{|c|}{ Pairwise P-values (D-in-D) } \\
\hline & $\underline{\mathrm{UC}}$ & SWCM & Website & $\begin{array}{l}\text { SWCM vs } \\
\text { UC }\end{array}$ & $\begin{array}{l}\frac{\text { Website }}{\text { vs UC }} \\
\end{array}$ & $\begin{array}{l}\text { Website } \\
\text { vs SWCM }\end{array}$ \\
\hline 7-day & $60.2(1.4)$ & $58.8(1.5)$ & 60.7 (1.5) & & & \\
\hline 90-day & $62.1(1.5)$ & $59.5(1.5)$ & $62.7(1.5)$ & & & \\
\hline Change & $+1.9(1.5)$ & $+0.7(1.6)$ & $+2.0(2.2)$ & 0.55 & 0.99 & 0.55 \\
\hline
\end{tabular}

\begin{tabular}{|c|c|c|c|c|c|c|}
\hline \multicolumn{7}{|c|}{ PHQ-9 } \\
\hline & \multicolumn{3}{|c|}{ LS Means (SE) } & \multicolumn{3}{|c|}{ Pairwise P-values (D-in-D) } \\
\hline & $\underline{U C}$ & $\underline{\text { SWCM }}$ & Website & $\begin{array}{l}\text { SWCM vs } \\
\text { UC }\end{array}$ & $\begin{array}{l}\frac{\text { Website }}{\text { vs UC }} \\
\text { val }\end{array}$ & $\begin{array}{l}\text { Website } \\
\text { vs SWCM }\end{array}$ \\
\hline 7-day & $3.6(0.5)$ & $3.3(0.5)$ & $3.9(0.5)$ & & & \\
\hline 90-day & $2.7(0.5)$ & $2.3(0.5)$ & $3.0(0.5)$ & & & \\
\hline Change & $-0.9(0.5)$ & $-1.0(0.6)$ & $-0.9(0.6)$ & 0.91 & 0.97 & 0.94 \\
\hline
\end{tabular}

